\title{
Evaluation of Drug Adsorption to Membrane Filters under Biowaiver Test Conditions
}

\author{
Kevin Kiehm and Jennifer B. Dressman ${ }^{1}$ \\ Institute of Pharmaceutical Technology, J. W. Goethe University, Max von Laue-Str. 9, 60438 \\ Frankfurt am Main, Germany
}

\section{INTRODUCTION}

D issolution testing has become a highly valuable in vitro test performed for quality control and regulatory purposes (1). According to the biopharmaceutical classification system (BCS), dissolution testing can be used to waive in vivo bioequivalence studies (2), where it can be applied to replace expensive pharmacokinetic studies and is suitable for the approval of generic medicinal products. Biowaivers can be requested for highly soluble and highly permeable compounds (BCS Class 1) in immediate-release (IR) solid oral dosage forms, provided they exhibit rapid in vitro dissolution. According to the guideline, an IR drug product is considered rapidly dissolving when not less than $85 \%$ of the labeled amount of drug dissolves within 30 min using USP Apparatus 1 at $100 \mathrm{rpm}$ or USP Apparatus 2 at $50 \mathrm{rpm}$.

Commonly, dissolution samples are filtered before quantitative analysis to separate undissolved excipients from the dissolved drug. To validate the filtration process, filter adsorption studies should be performed during dissolution method development. Since the biowaiver process is subject to stringent restrictions, it is important to determine adsorption under the test conditions that are particular to the biowaiver.

Lindenberg et al. (3) recently reported that not all filter materials are suitable for dissolution testing. Therefore, the recovery of a drug from the analyte should always be validated, since this is the only way to rule out adsorption to the filter. The aim of this study was to expand the adsorption study to a larger range of filters. Additionally, we evaluated the influence of soluble residues from filter materials on the drug determination by UV spectroscopy. Ten filter materials from three different filter suppliers were chosen. The adsorption of the model drugs acetylsalicylic acid and prednisolone in three different buffers was evaluated. Acetylsalicylic acid was chosen since it is a hydrophilic drug administered in a high dose, while prednisolone was chosen because it is lipophilic and is administered in a low dose. Furthermore, the buffers used in this study are the recommended media for biowaiver requests (Simulated Gastric Fluid USP without enzymes, a pH 4.5 buffer, and Simulated Intestinal Fluid USP without enzymes).

${ }^{1}$ Corresponding author.

\section{MATERIALS AND METHODS \\ Materials}

All chemicals including the model drugs acetylsalicylic acid and prednisolone were analytical grade and purchased commercially. Ten different filters from various filter manufactures were tested. Detailed information on the filters is shown in Table 1.

\section{Media}

The media used for the filtration experiments were $\mathrm{pH}$ 1.2 Simulated Gastric Fluid without pepsin $\left(\mathrm{SGF}_{\mathrm{sp}}\right), \mathrm{a}$ $\mathrm{pH} 4.5$ acetate buffer $(50 \mathrm{mM})$, and $\mathrm{pH} 6.8$ Simulated Intestinal Fluid without pancreatin $\left(\mathrm{SIF}_{\mathrm{sp}}\right)$, which are all USP Test Solutions (4). Stock solutions of the model drugs were prepared in each buffer solution; the concentrations are displayed in Table 2. Concentrations were chosen to simulate the $100 \%$ dissolution rate from the lowest standard dosage forms (100 mg acetylsalicylic acid and $5 \mathrm{mg}$ prednisolone) in their compendial dissolution volumes $(500 \mathrm{~mL}$ for acetylsalicylic acid and $900 \mathrm{~mL}$ for prednisolone) (4).

\section{Filtration Procedure}

Filtration studies were conducted in a manner similar to those described by Lindenberg et al. (3). The prepared

\begin{tabular}{ll}
\hline \multicolumn{2}{l}{ Table 1. Filters Used } \\
\hline Filter & Product Name \\
\hline Pall NL & Pall Acrodisc $0.45 \mu \mathrm{m}$ Nylon \\
\hline Pall PVDF & Pall Acrodisc $0.45 \mu \mathrm{m}$ PTFE \\
\hline Pall PTFE & Pall Acrodisc $0.45 \mu \mathrm{m}$ PVDF \\
\hline Pall GHP & Pall Acrodisc $0.45 \mu \mathrm{m}$ GHP \\
\hline Millipore NL & Millipore $0.45 \mu \mathrm{m}$ Nylon \\
\hline Millipore PVDF & Millipore $0.45 \mu \mathrm{m}$ PVDF \\
\hline Millipore PTFE & Millipore $0.45 \mu \mathrm{m}$ PTFE \\
\hline Roby NL & Whatman Roby 25/0.45 NL Polyamide/Nylon \\
\hline Roby GF & Whatman Roby 25/GF 92 Glass Fiber 1.0 $\mu \mathrm{m}$ \\
\hline Roby RC & Whatman Roby 25/0.45 RC Regenerated Cellulose \\
\hline
\end{tabular}


Table 2. Drug Concentrations in the Media ( $\mathrm{mg} / \mathrm{L}$ )

\begin{tabular}{lccc}
\hline & $\begin{array}{c}\mathbf{S G F}_{\mathbf{s p}} \\
\mathbf{p H ~ 1 . 2}\end{array}$ & $\begin{array}{c}\text { Acetate buffer } \\
\mathbf{p H ~ 4 . 5}\end{array}$ & $\begin{array}{c}\mathbf{S I F}_{\mathbf{s p}} \\
\mathbf{p H ~ 6 . 8}\end{array}$ \\
\hline Acetylsalicylic acid & 48.6 & 49.2 & 49.1 \\
\hline Prednisolone & 3.8 & 3.9 & 3.8 \\
\hline
\end{tabular}

stock solutions were filtered, and the recovery of drug in the filtrate was determined. Additionally, the recovery of the model drug was determined after the first $2 \mathrm{~mL}$ of filtrate was discarded. As a reference, the UV absorbance of the unfiltered stock solution was measured. Because filter materials may contain soluble residues as a result of the filter manufacturing process, the blank buffers were filtered, and the UV absorbance of each blank buffer filtrate was measured at the same wavelengths used for the determination of the model drugs.

Six samples of each drug-medium combination were drawn up into a glass syringe (Fortuna Optima ${ }^{\circledR}$ Luer Lock, Wertheim, Germany) and filtered immediately. The drug concentration in the filtrate was determined by UV spectroscopy. A new filter was used for each of the six experiments.

The drug recovery was calculated using the following equation:

$$
\text { Recovery }=\frac{A_{\text {filtrate }}-A_{\text {blank }}}{A_{\text {reference }}}
$$

where $A_{\text {filtrate }}$ is the absorbance of the filtered stock solution, $A_{\text {blank }}$ is the absorbance of the filtered blank buffer, and $A_{\text {reference }}$ is the absorbance of the unfiltered stock solution (reference).

\section{UV Analysis}

The UV system was a Hitachi U-3000 spectrophotometer (Tokyo, Japan). The program used for the analysis was UV Solutions 1.2. Acetylsalicylic acid was detected at $270 \mathrm{~nm}$, while prednisolone was detected at $247 \mathrm{~nm}$. The results are shown as mean values \pm SD.

A recovery of $>95 \%$ was selected as the criterion for acceptable adsorption behavior. A UV absorbance $<0.05$ was selected as the criterion for acceptable soluble residues. Values that did not meet the criteria are highlighted in the Tables.

Additionally, the blank buffer filtrates were measured by photon correlation spectroscopy (PCS) using a Malvern Zetasizer 3000HSA (Malvern Instruments Ltd., Malvern, UK) to investigate whether insoluble residues were causing UV absorbance due to light scattering.

\section{RESULTS}

\section{Blank Buffer UV Absorbance after Filtration}

Table 3 displays the UV absorbances of the blank buffers after filtration. None of the blank filtrates exhibited an absorbance $>0.05$ at the wavelength for acetylsalicylic acid. However, some showed a higher absorbance at the wavelength used to detect prednisolone. The absorbances for buffers filtered through the Pall GHP, the Roby Polyamide, Glass Fiber, and Regenerated Cellulose were higher than those observed for unfiltered buffer solutions. When the first $2 \mathrm{~mL}$ of filtrate was discarded before collecting samples for analysis, the absorbance was unchanged.

The somewhat different results obtained for the two drugs can be attributed to the different wavelengths used for analysis. Prednisolone was analyzed at a lower wavelength than acetylsalicylic acid. Additional

Table 3. UV Absorbance of Filter Material Residuals in the Blank Buffers at the Wavelengths Used for Determination of the Model Drugs

\begin{tabular}{|c|c|c|c|c|c|c|}
\hline \multirow[b]{2}{*}{ Filter } & \multicolumn{3}{|c|}{243 nm } & \multicolumn{3}{|c|}{$270 \mathrm{~nm}$} \\
\hline & pH 1.2 & pH 4.5 & pH 6.8 & pH 1.2 & pH 4.5 & pH 6.8 \\
\hline Pall NL & $0.00 \pm 0.00$ & $0.01 \pm 0.00$ & $0.02 \pm 0.00$ & $0.00 \pm 0.00$ & $0.00 \pm 0.00$ & $0.00 \pm 0.00$ \\
\hline Pall PVDF & $0.01 \pm 0.00$ & $0.00 \pm 0.00$ & $0.00 \pm 0.00$ & $0.00 \pm 0.00$ & $0.00 \pm 0.00$ & $0.00 \pm 0.00$ \\
\hline Pall PTFE & $0.00 \pm 0.00$ & $0.01 \pm 0.00$ & $0.00 \pm 0.00$ & $0.00 \pm 0.00$ & $0.00 \pm 0.00$ & $0.00 \pm 0.00$ \\
\hline Pall GHP & $0.13 \pm 0.02$ & $0.11 \pm 0.00$ & $0.09 \pm 0.00$ & $0.02 \pm 0.00$ & $0.02 \pm 0.00$ & $0.00 \pm 0.00$ \\
\hline Millipore NL & $0.02 \pm 0.00$ & $0.01 \pm 0.00$ & $0.00 \pm 0.00$ & $0.01 \pm 0.00$ & $0.01 \pm 0.00$ & $0.01 \pm 0.00$ \\
\hline Millipore PVDF & $0.00 \pm 0.00$ & $0.00 \pm 0.00$ & $0.00 \pm 0.00$ & $0.00 \pm 0.00$ & $0.00 \pm 0.00$ & $0.00 \pm 0.00$ \\
\hline Millipore PTFE & $0.00 \pm 0.00$ & $0.00 \pm 0.00$ & $0.00 \pm 0.00$ & $0.00 \pm 0.00$ & $0.00 \pm 0.00$ & $0.00 \pm 0.00$ \\
\hline Roby NL & $0.10 \pm 0.01$ & $0.10 \pm 0.01$ & $0.08 \pm 0.01$ & $0.04 \pm 0.01$ & $0.05 \pm 0.01$ & $0.03 \pm 0.00$ \\
\hline Roby GF & $0.06 \pm 0.01$ & $0.05 \pm 0.01$ & $0.04 \pm 0.01$ & $0.02 \pm 0.01$ & $0.02 \pm 0.00$ & $0.01 \pm 0.00$ \\
\hline Roby RC & $0.09 \pm 0.01$ & $0.06 \pm 0.01$ & $0.06 \pm 0.01$ & $0.02 \pm 0.00$ & $0.02 \pm 0.00$ & $0.02 \pm 0.00$ \\
\hline
\end{tabular}


Table 4. Recovery of Acetylsalicylic Acid (\%)

\begin{tabular}{|c|c|c|c|c|c|c|}
\hline \multirow[b]{2}{*}{ Filter } & \multicolumn{3}{|c|}{ no medium discarded } & \multicolumn{3}{|c|}{$2 \mathrm{~mL}$ medium discarded } \\
\hline & pH 1.2 & pH 4.5 & pH 6.8 & pH 1.2 & pH 4.5 & pH 6.8 \\
\hline Pall NL & $80.4 \pm 3.1$ & $97.3 \pm 0.9$ & $96.5 \pm 1.4$ & $98.3 \pm 1.7$ & $99.1 \pm 0.8$ & $100.7 \pm 1.0$ \\
\hline Pall PVDF & $91.9 \pm 1.8$ & $99.4 \pm 1.1$ & $92.9 \pm 1.6$ & $97.6 \pm 0.8$ & $99.2 \pm 1.0$ & $97.6 \pm 0.7$ \\
\hline Pall PTFE & $98.9 \pm 0.8$ & $98.9 \pm 0.6$ & $100.9 \pm 0.8$ & $99.6 \pm 1.1$ & $99.8 \pm 0.6$ & $100.6 \pm 0.8$ \\
\hline Pall GHP & $94.0 \pm 1.0$ & $99.9 \pm 1.5$ & $100.0 \pm 1.5$ & $101.0 \pm 1.2$ & $99.8 \pm 0.6$ & $101.9 \pm 2.3$ \\
\hline Millipore NL & $77.6 \pm 2.6$ & $93.6 \pm 1.5$ & $86.7 \pm 0.9$ & $96.2 \pm 0.9$ & $99.1 \pm 0.5$ & $99.9 \pm 0.9$ \\
\hline Millipore PVDF & $99.6 \pm 0.8$ & $99.5 \pm 0.8$ & $99.9 \pm 0.7$ & $99.8 \pm 0.9$ & $100.2 \pm 0.3$ & $99.9 \pm 0.9$ \\
\hline Millipore PTFE & $100.3 \pm 0.8$ & $100.2 \pm 1.8$ & $99.6 \pm 0.4$ & $99.9 \pm 1.0$ & $99.7 \pm 1.0$ & $100.2 \pm 0.8$ \\
\hline Roby NL & $82.1 \pm 1.9$ & $96.7 \pm 1.6$ & $93.6 \pm 1.6$ & $98.3 \pm 1.1$ & $100.0 \pm 0.5$ & $99.5 \pm 0.5$ \\
\hline Roby GF & $100.4 \pm 2.2$ & $100.3 \pm 1.6$ & $100.0 \pm 1.6$ & $99.1 \pm 0.6$ & $99.6 \pm 1.2$ & $99.8 \pm 0.7$ \\
\hline Roby RC & $99.0 \pm 2.7$ & $100.9 \pm 1.1$ & $97.3 \pm 0.9$ & $101.1 \pm 2.4$ & $100.0 \pm 1.5$ & $100.1 \pm 0.9$ \\
\hline
\end{tabular}

experiments confirmed that filter contributions to absorbance declined with an increase in analytical wavelength. To rule out the possibility that undissolved particles were responsible for the appearance of a UV absorbance, the samples were analyzed using photon correlation spectroscopy (PCS). No particles were detected. So it was assumed that some soluble residues were extracted by the aqueous buffer solutions during filtration. No effects of buffer composition and $\mathrm{pH}$ on the extraction of soluble residues were observed.

\section{Drug Recovery}

The results for acetylsalicylic acid are listed in Table 4. As seen in Figure 1A, acceptable results were obtained for the recovery of acetysalicylic acid with most of the filter/buffer combinations, with the exception of the nylon/polyamide filters (from all manufacturers) and the PVDF and GHP filters from Pall. These did not meet the criterion of a $95 \%$ drug recovery in the filtrate. Figure 1B displays the recovery of acetylsalicylic acid after the first $2 \mathrm{~mL}$ of media was discarded. When $2 \mathrm{~mL}$ was discarded before analysis, the recovery improved, and all filters in all buffer media exhibited acceptable results (drug recovery $>95 \%$ ).

The results for prednisolone are summarized in Table 5. Figure $2 \mathrm{~A}$ displays the recovery of prednisolone without discarding $2 \mathrm{~mL}$, while Figure $2 \mathrm{~B}$ shows the drug recovery after discarding $2 \mathrm{~mL}$ of filtrate before analysis.

In general, results were lower for prednisolone recovery. Without discarding $2 \mathrm{~mL}$ of medium before collecting samples for analysis, acceptable recovery (>95\%) was only obtained with the PVDF and PTFE filters from Millipore, the GHP filters from Pall, and the Glass Fiber and RC filters from Whatman Schleicher \& Schuell. The highest recoveries were found for the Millipore PVDF and PTFE
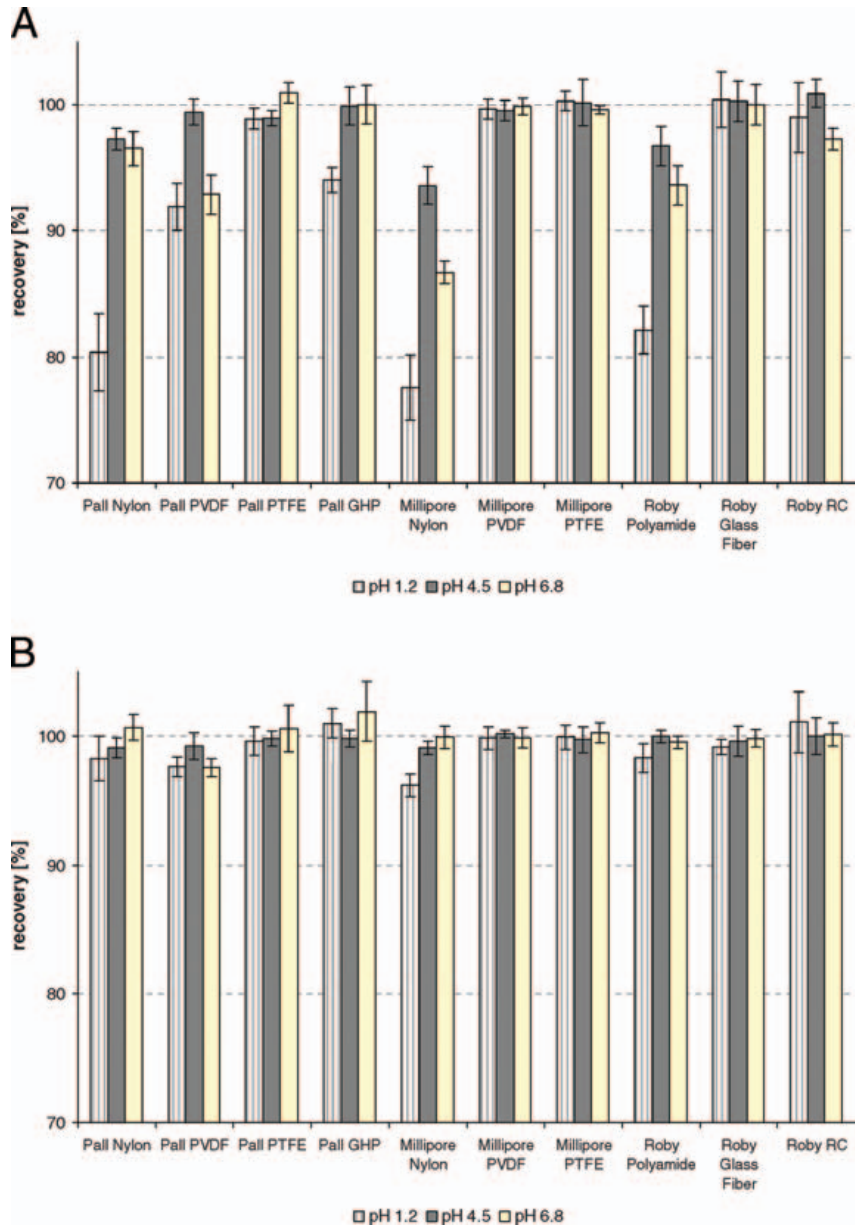

Figure 1. (A) Recovery of acetylsalicylic acid without discarding the first $2 \mathrm{~mL}$ of filtrate. (B) Figure 1b. Recovery of acetylsalicylic acid after the discard of $2 m L$ media before analysis. 


\begin{tabular}{|c|c|c|c|c|c|c|}
\hline \multirow[b]{2}{*}{ Filter } & \multicolumn{3}{|c|}{ no medium discarded } & \multicolumn{3}{|c|}{$2 \mathrm{~mL}$ medium discarded } \\
\hline & pH 1.2 & pH 4.5 & pH 6.8 & pH 1.2 & pH 4.5 & pH 6.8 \\
\hline Pall NL & $85.8 \pm 2.8$ & $74.5 \pm 2.0$ & $65.3 \pm 2.5$ & $98.2 \pm 0.4$ & $92.9 \pm 0.9$ & $90.9 \pm 1.8$ \\
\hline Pall PVDF & $88.9 \pm 4.3$ & $96.0 \pm 1.1$ & $93.0 \pm 1.6$ & $97.2 \pm 0.9$ & $98.6 \pm 0.8$ & $97.8 \pm 1.0$ \\
\hline Pall PTFE & $86.3 \pm 2.0$ & $84.2 \pm 1.4$ & $83.4 \pm 1.9$ & $98.6 \pm 0.4$ & $98.2 \pm 1.5$ & $96.3 \pm 1.0$ \\
\hline Pall GHP & $96.7 \pm 3.7$ & $96.8 \pm 0.8$ & $101.7 \pm 3.0$ & $100.1 \pm 2.0$ & $97.5 \pm 2.0$ & $103.0 \pm 2.0$ \\
\hline Millipore NL & $74.0 \pm 2.1$ & $55.1 \pm 3.7$ & $54.3 \pm 4.4$ & $99.7 \pm 0.5$ & $90.9 \pm 2.1$ & $91.4 \pm 1.3$ \\
\hline Millipore PVDF & $99.2 \pm 1.9$ & $99.2 \pm 0.9$ & $99.0 \pm 0.9$ & $99.7 \pm 1.3$ & $99.4 \pm 0.6$ & $99.7 \pm 0.8$ \\
\hline Millipore PTFE & $99.7 \pm 1.8$ & $98.4 \pm 2.3$ & $101.4 \pm 1.1$ & $100.4 \pm 0.7$ & $98.5 \pm 2.1$ & $101.9 \pm 2.9$ \\
\hline Roby NL & $83.3 \pm 1.0$ & $69.7 \pm 5.3$ & $68.0 \pm 2.6$ & $97.4 \pm 1.0$ & $90.3 \pm 1.9$ & $90.7 \pm 2.1$ \\
\hline Roby GF & $100.3 \pm 2.8$ & $97.9 \pm 4.2$ & $97.7 \pm 3.8$ & $98.6 \pm 1.3$ & $98.1 \pm 1.7$ & $99.5 \pm 1.6$ \\
\hline Roby RC & $101.7 \pm 1.1$ & $102.4 \pm 4.0$ & $100.1 \pm 2.5$ & $98.6 \pm 1.3$ & $96.9 \pm 1.1$ & $97.1 \pm 3.4$ \\
\hline
\end{tabular}

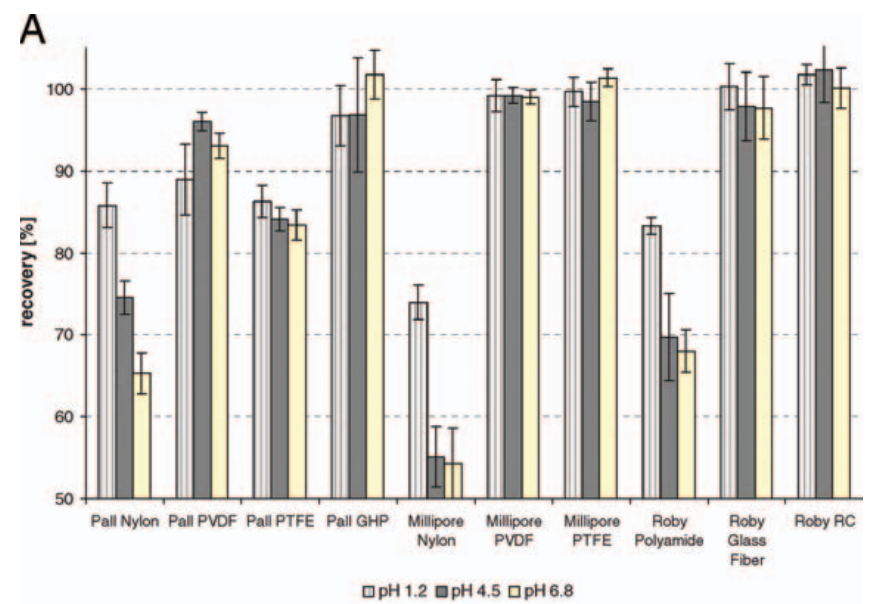

B

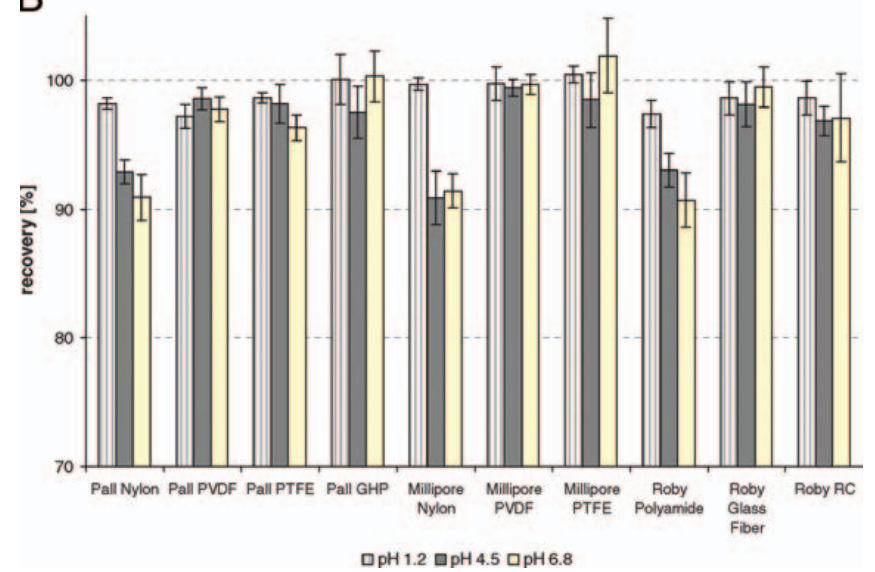

Figure 2. (A) Recovery of prednisolone without discarding the first $2 \mathrm{~mL}$ of filtrate. (B) Recovery of prednisolone after the discard of $2 \mathrm{~mL}$ media before analysis.

Dissolution Technologies | NOVEMBER 2008 and the Roby Regenerated Cellulose filters. The nylon/ polyamide filters exhibited the poorest recovery.

After the first $2 \mathrm{~mL}$ of sample media was discarded before analysis, the nylon/polyamide filters (all manufacturers) failed to meet the $95 \%$ recovery criterion for prednisolone.

An explanation for the higher drug loss due to adsorption to the filter material could be the lower concentration of prednisolone in the buffer media compared with acetylsalicylic acid. Additionally, prednisolone is more lipophilic than acetylsalicylic acid, and lipophilicity is generally assumed to facilitate adsorption (5).

Generally, buffer composition and $\mathrm{pH}$ did not influence the drug recovery for the different filter materials. However, a pH-dependent adsorption was only observed for the nylon filters. For acetylsalicylic acid, a lower recovery was found at $\mathrm{pH} 1.2$, while for prednisolone, a lower recovery was observed at higher $\mathrm{pH}$ values.

\section{DISCUSSION}

There was minimal adsorption of acetylsalicylic acid and prednisolone on most of the investigated filter materials. When the first $2 \mathrm{~mL}$ of filtrate was discarded before analysis, most of the tested filter materials showed a recovery $>95 \%$ and should therefore be suitable for dissolution testing in the biowaiver buffers. Under these conditions, unacceptable drug loss due to adsorption was only observed for the nylon/polyamide material. With respect to both chosen model drugs prednisolone and acetylsalicylic acid, the results indicate that the nylon/ polyamide material is unacceptable for dissolution testing. The high adsorption property of nylon has been reported 
by other authors $(3,6)$. Therefore, other filter materials should be used for dissolution experiments.

In contrast to the results obtained by Lindenberg et al., discarding the first $2 \mathrm{~mL}$ of filtrate before analysis improved the recovery of the model drugs. After the available active adsorption sites are saturated, the subsequent filtration process does not further decrease the drug concentration in the filtrate.

An additional benefit is that soluble residuals that could be present from the manufacturing process are eliminated by discarding the first $2 \mathrm{~mL}$. Without discard of $2 \mathrm{~mL}$ before analysis, the UV absorbance of the soluble residues could result in a higher cumulative absorbance and thus an overestimate of drug recovery.

In summary, the studies indicate that drug recovery should always be validated for a specific filter material, since the absence of filter adsorption and soluble residuals cannot otherwise be assured.

\section{REFERENCES}

1. Dissolution Testing of Immediate Release Solid Oral Dosage Forms; Guidance for Industry; U.S. Department of Health and Human Services, Food and Drug Administration, Center for Drug Evaluation and Research, U.S. Government Printing Office: Washington, DC, 1997.

2. Waiver of In Vivo Bioavailability and Bioequivalence Studies for Immediate-Release Solid Dosage Forms Based on a Biopharmaceutics Classification System; Guidance for Industry; U.S. Department of Health and Human Services, Food and Drug Administration, Center for Drug Evaluation and Research, U.S. Government Printing Office: Washington, DC, 2000.

3. Lindenberg, M.; Wiegand, C.; Dressman, J. B. Comparison of the adsorption of several drugs to typical filter materials. Dissolution Technol. 2005, 12 (1), 22-25.

4. United States Pharmacopeia and National Formulary USP 31-NF 26; The United States Pharmacopeial Convention, Inc.: Rockville, MD, 2008.
5. Unger, J. K.; Kuehlein, G.; Schroers, A.; Gerlach, J. C.; Rossaint, R. Adsorption of xenobiotics to plastic tubing incorporated into dynamic in vitro systems used in pharmacological research-limits and progress. Biomaterials 2001, 22 (14), 2031-2037.

6. Guilfoyle, D. E.; Roos, R.; Carito, S. L. An evaluation of preservative adsorption onto nylon membrane filters. J. Parent. Sci. Technol. 1990, 44 (6), 314-319. 